\title{
SPATIAL DISTRIBUTIONS OF YOUNG STARS
}

\author{
Adam L. Kraus and Lynne A. Hillenbrand \\ California Institute of Technology, Department of Astrophysics, MC 105-24, Pasadena, CA 91125; alk@astro.caltech.edu \\ Received 2008 April 29; accepted 2008 September 4; published 2008 September 16
}

\begin{abstract}
We analyze the spatial distribution of young stars in Taurus-Auriga and Upper Sco, as determined from the two-point correlation function (i.e., the mean surface density of neighbors). The corresponding power-law fits allow us to determine the fractal dimensions of each association's spatial distribution, measure the stellar velocity dispersions, and distinguish between the bound binary population and chance alignments of members. We find that the fractal dimension of Taurus is $D \sim 1.05$, consistent with its filamentary structure. The fractal dimension of Upper Sco may be even shallower $(D \sim 0.7)$, but this fit is uncertain due to the limited area and possible spatially variable incompleteness. We also find that random stellar motions have erased all primordial structure on scales of $\leq 0.07^{\circ}$ in Taurus and $\leq 1.7^{\circ}$ in Upper Sco; given ages of $\sim 1$ and $\sim 5 \mathrm{Myr}$, the corresponding internal velocity dispersions are $\sim 0.2$ and $\sim 1.0 \mathrm{~km} \mathrm{~s}^{-1}$, respectively. Finally, we find that binaries can be distinguished from chance alignments at separations of $\$ 120^{\prime \prime}(17,000 \mathrm{AU})$ in Taurus and $\$ 75^{\prime \prime}(11,000 \mathrm{AU})$ in Upper Sco. The binary populations in these associations that we previously studied, spanning separations of $3^{\prime \prime}-30^{\prime \prime}$, is dominated by binary systems. However, the few lowest mass pairs $\left(M_{\text {prim }} \leq 0.3 M_{\odot}\right)$ might be chance alignments.

Subject headings: binaries: visual — methods: statistical — stars: formation — stars: kinematics — stars: pre-main-sequence - stars: statistics

Online material: color figures
\end{abstract}

\section{INTRODUCTION}

The spatial distribution of young stars is a powerful diagnostic of their formation and early evolution. Young stars trace the gas distribution from which they formed, so the large-scale structure of a young association retains these primordial features after the gas has been accreted or dispersed. On intermediate scales, the absence of structure indicates the typical distance over which stars have randomly dispersed since their birth, and therefore the velocity dispersion for the association. Finally, the enhanced stellar density on small scales outlines the binary population, distinguishing bound binary systems from chance alignments between young stars. Some of these topics have been addressed in previous work on young star distributions (Gomez et al. 1993; Larson 1995; Simon 1997; Bate et al. 1998; Hartmann 2002), but the modern census of several key star-forming regions is more complete and extends to lower masses than a decade ago, so the analysis is worth revisiting.

The traditional tool for studying spatial distributions is the two-point correlation function (TPCF). The TPCF, $w(\theta)$, is defined as the number of excess pairs of objects with a given separation $\theta$ over the expected number for a random distribution (Peebles 1980). The TPCF is proportional to the mean surface density of neighbors, so it is often recast in terms of this more intuitive quantity: $\Sigma(\theta)=\left(N_{*} / A\right)[1+w(\theta)]$, where $A$ is the survey area and $N_{*}$ is the total number of stars.

In this Letter, we describe an updated relation for $\Sigma(\theta)$ in Taurus and present the first such analysis for Upper Sco, then we fit power laws for the different angular regimes. Finally, we interpret our results to address three questions: What is the primordial fractal dimension of star-forming regions, and how does it relate to their observed geometry? What is the primordial velocity dispersion suggested by each association's randomization? And what is a wide binary companion, and can it be distinguished from an unbound association member?

\section{THE CORRELATION FUNCTIONS OF TAURUS AND UPPER SCO}

We compiled our Taurus sample from the member list in Kraus \& Hillenbrand (2007a, 2008), plus the Class 0/I sources that were compiled by Kenyon \& Hartmann (1995). We omitted the latter sources from our multiplicity surveys because their stellar properties are uncertain, but we include them here because that information is not necessary for clustering analysis. We have also included the partial list of new sources identified in data from the Taurus Spitzer Legacy Project (Padgett et al. 2006) as described by Luhman et al. (2006). For separations of $<30^{\prime \prime}$, we have calculated the surface density of neighbors only among those sources included in our initial wide binary survey. We have neglected the Class $0 / I$ and heavily embedded sources because only some have been surveyed for multiplicity in the mid-infrared wavelengths (e.g., Duchêne et al. 2004), and not with uniform sensitivity. Our full sample consists of 272 members, while the binary-regime sample consists of 226. The Taurus sample is almost certainly incomplete, as a number of additional candidates have been identified in the Taurus Legacy Project (Padgett et al. 2006) and the XEST survey (Scelsi et al. 2007). However, preliminary reports suggest an increment of $\$ 20 \%$ in the total sample. Even if these new members do not trace the known distribution, their influence should be modest.

The census of Upper Sco across the full association is very incomplete, so we implemented our analysis for intermediate and large separations $\left(\theta>30^{\prime \prime}\right)$ using only members in two heavily studied fields observed by Preibisch et al. (2002), the $2 \mathrm{dfE}$ and $2 \mathrm{dfW}$ fields. The census of members in these fields is not complete, but we expect that it is the least incomplete. As for Taurus, we calculated the surface density of neighbors at $<30^{\prime \prime}$ using the full sample of our wide binary surveys; this choice maximizes our sample size for small separations (where the statistics are weakest). The $2 \mathrm{dfE} / 2 \mathrm{dfW}$ and binary samples consist of 162 and 352 members.

In Figure 1, we plot the locations of our sample members 

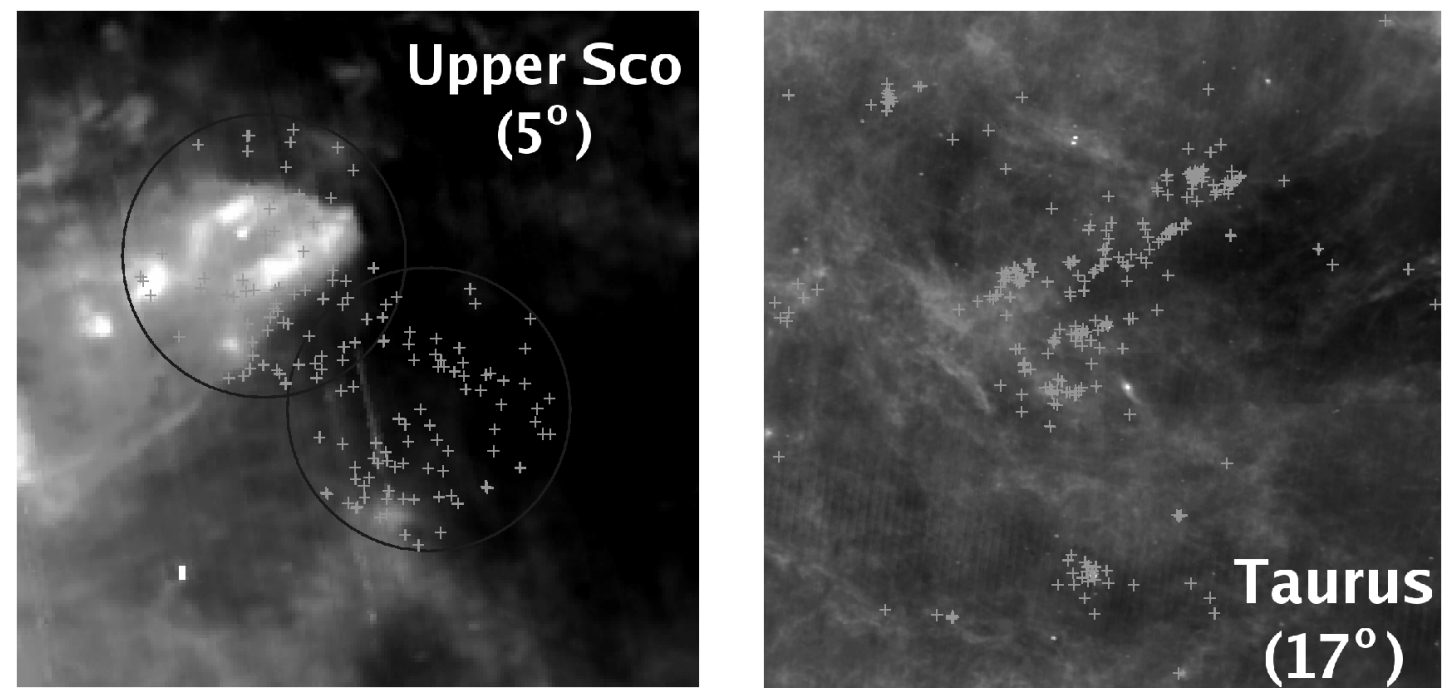

FIG. 1.-Locations of stars in Taurus and Upper Sco, superimposed on $60 \mu \mathrm{m}$ IRAS images. Members are denoted by green crosses, while the sample fields in Upper Sco are denoted by blue circles. The field of view is $17^{\circ}$ in Taurus and $5^{\circ}$ in Upper Sco. Known members in Upper Sco outline the dusty clouds in the northern field, suggesting systematic incompleteness for extincted members. [See the electronic edition of the Journal for a color version of this figure.]

superimposed on archival $60 \mu \mathrm{m}$ IRAS images. In Upper Sco, we see evidence of incompleteness for the northern field. Most of the known members outline the dusty regions, suggesting that any members in these regions were too extincted to have been identified. As we discuss later, this could affect the TPCF on scales of $\geq 1^{\circ}$. In Taurus, the distribution traces the fila-
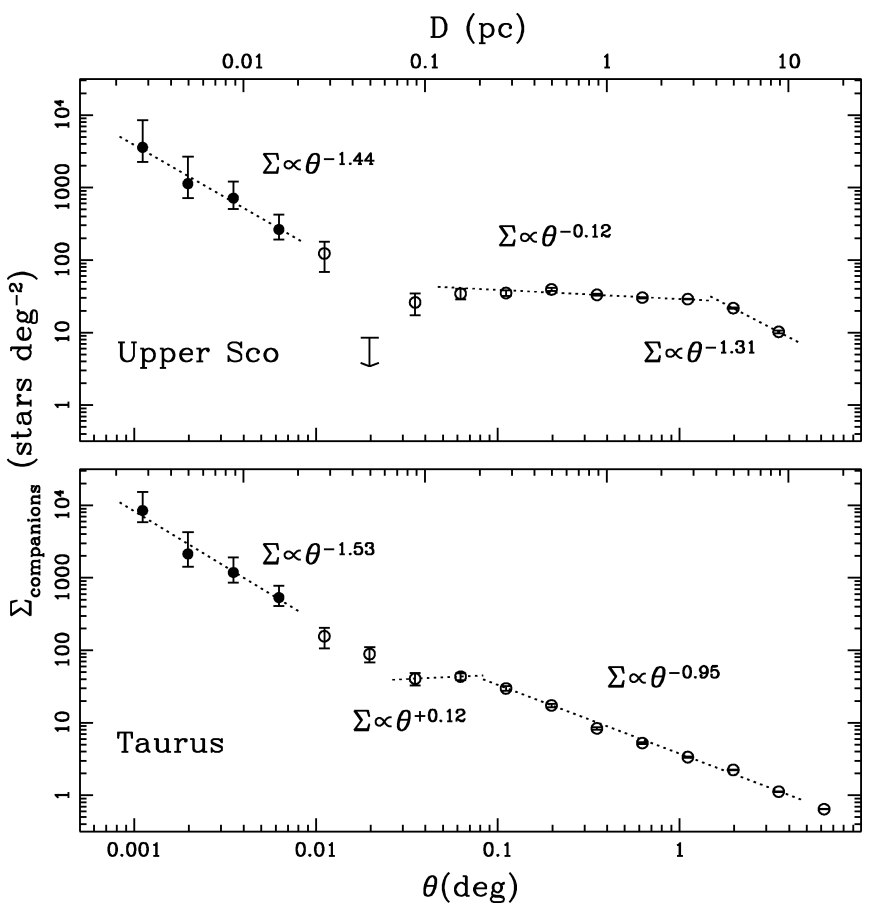

FIG. 2.-Two-point correlation functions for members of Upper Sco and Taurus. These plots show the surface density of neighbors as a function of separation, $\Sigma(\theta)$, with $\theta$ in degrees (bottom axis) or in parsecs (top axis). The observations are from our recent wide binary survey (Kraus \& Hillenbrand 2008; filled circles) or membership surveys in the literature (open circles). For each association, we have fit power laws to the small-scale regime (red; binary systems), the large-scale regime (blue; association members distributed according to the primordial structure), and the intermediate regime (green; association members with a randomized spatial distribution). [See the electronic edition of the Journal for a color version of this figure.] mentary dust, although there are also many filaments that do not include any known members.

We directly measured $\Sigma(\theta)$ for Taurus because our sample spans the entire area of the association. However, for bounded subsets (as in Upper Sco), it is often easier to evaluate the TPCF via a Monte Carlo-based definition, $w(\theta)=$ $N_{p}(\theta) / N_{r}(\theta)-1$, where $N_{p}(\theta)$ is the number of pairs with separations in a bin centered on $\theta$ and $N_{r}(\theta)$ is the expected number of pairs for a random distribution of objects over the bounded area (Hewett 1982). The advantage is that this method does not require edge corrections, unlike direct measurement of $\Sigma(\theta)$. In both cases, we report our results as $\Sigma(\theta)$ since it is a more visually motivated quantity than $w(\theta)$. In Figure 2, we plot $\Sigma(\theta)$ for Upper Sco (top) and Taurus (bottom) spanning a separation range of $3^{\prime \prime}$ to $10^{\circ}$.

Based on the predicted time evolution of young associations (Bate et al. 1998), we expect that $\Sigma(\theta)$ can be fit with a twicebroken power law, corresponding to structure on three scales. At small scales, bound binary systems yield a relatively steep power law. At large scales (and for young ages, $<1$ crossing time), intra-association clustering yields a shallower (but nonzero) power law that corresponds to the primordial structure of the association. Finally, at intermediate separations, the random motion of association members acts to smooth out the primordial structure and yield a constant surface density (and thus a slope near zero, according to the simulations of Bate et al. 1998). The first knee (transition between gravitationally bound multiplicity and a smooth randomized distribution) corresponds to the maximum angular scale for distinguishing binary systems, while the second knee (transition between a random distribution and primordial structure) corresponds to an angular scale that depends on the age since members were released from their natal gas clouds, $\tau$, and the internal velocity dispersion, $v_{\text {int }}$, where $\theta \propto \tau v_{\text {int }}$. Hartmann (2002) suggested that this break also could indicate the mean spacing of cores along filaments (the Jeans length), which assumes that stars have randomized by a smaller angular scale and that the inferred value characteristic angular scale, the inferred value of $v_{\text {int }}$ is an upper limit.

In Table 1, we summarize our weighted least-squares fits for 
TABLE 1

POWER LAW FITS

\begin{tabular}{|c|c|c|c|}
\hline Regime & Sep Range ${ }^{a}$ & $\alpha$ & $C\left(\log \operatorname{deg}^{-2}\right)$ \\
\hline \multicolumn{4}{|c|}{ Upper Sco } \\
\hline Binat & $3^{\prime \prime}-30^{\prime \prime}$ & $-1.44 \pm 0.41$ & $2.98 \pm 0.12$ (at $\left.9.5^{\prime \prime}\right)$ \\
\hline Intermec & $2.8^{\prime}-1.5^{\circ}$ & $-0.12 \pm 0.02$ & $1.537 \pm 0.010\left(\right.$ at $\left.16^{\prime}\right)$ \\
\hline Association & $1.5^{\circ}-4.7^{\circ}$ & $-1.31 \pm 0.09$ & $1.174 \pm 0.011\left(\right.$ at $\left.2.6^{\circ}\right)$ \\
\hline \multicolumn{4}{|c|}{ Taurus } \\
\hline Binary & $3^{\prime \prime}-30^{\prime \prime}$ & $-1.53 \pm$ & $3.28 \pm 0$ \\
\hline Interme & $1.6^{\prime}-5.0^{\prime}$ & $0.12 \pm 0.39$ & $1.62 \pm 0.05\left(\right.$ at $\left.2.8^{\prime}\right)$ \\
\hline Association . & $5.0^{\prime}-4.7^{\circ}$ & $-0.951 \pm 0.007$ & $0.650 \pm 0.005\left(\right.$ at $\left.1.2^{\circ}\right)$ \\
\hline
\end{tabular}

${ }^{a}$ There is a small range of separations between the binary and intermediate regimes where the data are consistent with our power-law fits, but the uncertainties are too large for those data to contribute meaningfully to the fits.

the power-law slope $\alpha$ and zero point $C$ in each regime. The binary regime was fit in the range probed in our survey of wide multiplicity $\left(3^{\prime \prime}-30^{\prime \prime}\right)$, while the intermediate and association regimes were fit in the ranges where the error bars were $\$ 3 \%$. We established the zero point of each fit at the logarithmic center of the angular range in order to minimize correlation between $\sigma_{\alpha}$ and $\sigma_{C}$. In Upper Sco, both the inner and middle power laws are clearly defined, but the fit for the outer regime is uncertain because the angular scale is similar to the size of the survey area $\left(\sim 2^{\circ}-4^{\circ}\right)$. In Taurus, the inner and outer power laws are clearly defined, but the fit for the intermediate regime is uncertain. The TPCF at separations of $2^{\prime}-4^{\prime}$ is flat and diverges from the fit for larger and smaller separations by 3-5 $\sigma$, so we provisionally assume that this separation range represents the intermediate regime. The points at smaller separations also fall below the projection of the association-regime power law, while the points at larger separations agree well with the overall fit, suggesting that our inferred value of $v_{\text {int }}$ is at most an upper limit. The locations of the first knee, where the two power laws are equal, are $\theta_{1, \mathrm{USco}} \sim 75^{\prime \prime}$ and $\theta_{1, \mathrm{Tau}} \sim 120^{\prime \prime}$; the respective locations of the second knee are $\theta_{2, \text { Usco }} \sim 1.7^{\circ}$ and $\theta_{2, \text { Tau }} \sim 0.07^{\circ}$. The formal uncertainties in these measurements are only $\sim 2 \%-3 \%$, but the errors are dominated by systematic uncertainties in the membership census and in the angular range over which to fit each regime.

\section{ASSOCIATION REGIME: THE FRACTAL DIMENSION OF TAURUS}

The primordial spatial distribution of young stars should trace the overdensities in the original gas distribution from which those stars formed (e.g., Hartmann 2002; Bate et al. 2003). Even if these gas distributions have dispersed, the remnants of primordial structure in the stellar distribution can still provide a key constraint to the distribution of overdensities during star formation. Early studies of TPCFs have suggested that current (and presumably primordial) stellar distributions are fractal in nature (e.g., Larson 1995; Simon 1997), with selfsimilar structure on a range of angular scales. Similar TPCFs can be reproduced (at least over a decade of separation) with simpler distributions like a finite number of nonfractal subclusters following a simple $r^{\alpha}$ profile (Bate et al. 1998). However, our TPCF for Taurus follows a single power law across $>2$ decades of separation, so it appears to be genuinely selfsimilar. The dimensionality $D$ of a fractal distribution indicates the extent to which it fills space, such that the number of neighbors $N$ within a distance $\theta$ goes as $N(\theta) \propto \theta^{D}$. This parameter is related to the surface density of neighbors; if $\Sigma(\theta) \propto \theta^{\alpha}$, then $D=\alpha+2$ (Larson 1995).

The fractal dimension is a result of the turbulent fragmen- tation that leads to star formation, and most models yield filamentary structure (i.e., a dimension near unity). As we showed in $\S 2$, the observed power-law slope for Taurus in the largescale regime is $\alpha=-0.951 \pm 0.007$, indicating that the fractal dimension $(D=1.049 \pm 0.007)$ is indeed close to unity. This result is consistent with visual inspection of the stellar distribution, as well as with $\mathrm{CO}$ maps of the remaining gas distribution (e.g., Goldsmith et al. 2008). Our value is significantly lower than the fractal dimension suggested by Larson (1995) and Simon (1997), $D=1.4$, but close to the more recent value suggested by Hartmann et al. (2002). Our sample is significantly more complete than the older samples; based on our reconstruction of those samples, most of the new (typically low-mass) members are located near the major concentrations (e.g., Strom \& Strom 1994; Briceño et al. 2002) rather than in the more distributed population (e.g., Slesnick et al. 2006). These members increase the surface density of neighbors at small separations, yielding a steeper slope for $\Sigma(\theta)$. However, the census is still incomplete $(\S 2)$ and if the incompleteness is spatially variable, such as for heavily embedded brown dwarfs, then our updated power-law slope could be incorrect.

We are hesitant to estimate the fractal dimension in Upper Sco. The appropriate regime in the TPCF includes only two separation bins, so the choice of bin locations could significantly affect the slope. Incompleteness in the dusty northern region could also influence the inferred large-scale structure. However, if we adopt our power-law fit from $\S 2(\alpha=-$ $1.31 \pm 0.09$ ), we find that $D=0.69 \pm 0.09$ on scales of $\sim 2^{\circ}$.

\section{INTERMEDIATE REGIME: THE PRIMORDIAL VELOCITY DISPERSION}

The angular scales over which structure has been randomized, as indicated by the location of the second knee in $\Sigma(\theta)$, directly constrains the primordial velocity dispersion for each association (e.g., Bate et al. 1998). This constraint is particularly important for low-density associations like Taurus and Upper Sco because the expected velocity dispersion ( $\$ 1-2 \mathrm{~km}$ $\mathrm{s}^{-1}$; Frink et al. 1997) may be too low to be measured easily via a direct method (like high-resolution spectroscopy to determine radial velocities). $\mathrm{OB}$ and $\mathrm{T}$ associations are not bound once their unaccreted gas is expelled (e.g., Lada et al. 1984), so the internal velocity dispersion is critical for determining how long they can persist as recognizable moving groups (like the $\beta$ Pic, TW Hya, or $\mu$ Oph associations; Webb et al. 1999; Zuckerman et al. 2004; Mamajek 2006) and how long substructure can remain in these moving groups.

Allowing for projection effects, the angular scales of each TPCF's outer knee correspond to physical dispersion scales of $\sim 0.23 \mathrm{pc}$ in Taurus and $\sim 6 \mathrm{pc}$ in Upper Sco. Given the characteristic ages of each association $(\sim 1 \mathrm{Myr}$ and $\sim 5 \mathrm{Myr}$, respectively), the corresponding characteristic velocity dispersions are $\sim 0.2$ and $\sim 1.0 \mathrm{~km} \mathrm{~s}^{-1}$. As we previously discussed, there is uncertainty in the fits, so these values should be taken with caution. We also note that these values represent the velocity dispersion with respect to other stars only within an angular distance of $\sim \theta_{\text {knee }}$. We cannot rule out the possibility that larger substructures are moving coherently with a higher velocity dispersion, only that any substructure with angular size $\theta$ is not moving with sufficient speed $(\dot{\theta} \sim \theta / \tau)$ that its angular displacement from birth is of order $\theta$. This limit also suggests an explanation for the larger velocity dispersion in Upper Sco; even if the velocity dispersion within $\sim 0.1-0.2$ pc substructures is the same as in Taurus, the observed TPCF could be repro- 
duced if the velocity dispersion between those substructures is $\sim 1 \mathrm{~km} \mathrm{~s}^{-1}$. A scale dependence in the velocity dispersion could also explain previous proper-motion studies in Taurus, which found velocity dispersions within the major subclumps (on scales of $\sim 1-3$ pc; e.g., Jones \& Herbig 1979) that were $\sim 1$ $\mathrm{km} \mathrm{s}^{-1}$.

A similar effect has been noted in locations like the ONC, where radial velocities show an overall north-south gradient of $\sim 5 \mathrm{~km} \mathrm{~s}^{-1}$ in addition to the local velocity dispersion of 2-3 $\mathrm{km} \mathrm{s}^{-1}$ (Fũrész et al. 2008). However, there is also observational evidence that small-scale velocity dispersions are higher in denser clusters; submillimeter observations of IRS 1 in NGC 2264 (Williams \& Garland 2002) found that six protostellar cores (spanning $0.44 \mathrm{pc}$ ) had a velocity dispersion of $0.9 \mathrm{~km}$ $\mathrm{s}^{-1}$, which much higher than the velocity dispersion that we find in Taurus, although also closer to the value for scales of 1-3 pc suggested by Jones \& Herbig (1979).

Our results suggest that regions like Taurus and Upper Sco are even less dynamically active, relative to the ONC, than their lower densities might imply. The velocity dispersions also provide a direct estimate of the virial velocity in the natal environment (before the removal of gas) and therefore jointly constrain the typical mass and size of a star-forming clump: $M v^{2} \sim\left(3 G M^{2}\right) /(5 R)$ or $M / R \sim\left(5 v^{2}\right) /(3 G)$, yielding $M / R \sim 15$ in Taurus and $M / R \sim 550$ in Upper Sco, where the mass is in solar masses and the radius is in parsecs. Thus, the primordial star-forming structures that are now dispersing with these characteristic velocities were smaller and/or more massive in Upper Sco than in Taurus.

\section{BINARY REGIME: WHAT IS A BINARY SYSTEM?}

The existence and properties of wide binary systems are critical for constraining multiple star formation in the limiting case of large separations and early times. If wide binaries form out of a single protostellar clump, then the maximum separation also constrains the maximum size of clumps that can collapse to become bound systems. As previous authors have suggested (e.g., Larson 1995), the outer edge of the young binary separation distribution is similar to the mean Jeans length for nearby molecular clouds. This limit is also similar to the maximum separation seen in the field (e.g., Duquennoy \& Mayor
1991), suggesting that some wide binaries join the field without being subjected to significant dynamical interactions. However, study of young binaries is complicated by the difficulty of distinguishing gravitationally bound binary pairs from coeval, comoving association members that are aligned in projection. We addressed this issue for a single system in Upper Sco (USco J1606-1935; Kraus \& Hillenbrand 2007b) by calculating the association's TPCF to determine the probability that it is a bound system; we now extend our analysis to the full known populations of Taurus and Upper Sco.

We find that the transition between the binary and intermediate regimes occurs at $\sim 11,000$ AU in Upper Sco and $\sim 17,000$ AU in Taurus. The binary population therefore extends at least to these angular scales, but we cannot distinguish binary companions from chance alignments outside this limit. The difference between these regimes is a result of the higher total wide binary frequency in Taurus (Kraus \& Hillenbrand 2007a, 2008), as the overall surface density of "contaminant" co-association members is similar in both associations. The number statistics do not support any assertions regarding the outer maximum limit of binary formation, but this angular scale matches both the maximum binary separation seen in the field and the typical Jeans length, so we do not expect to find many binary systems with wider separations.

Candidate companions inside this limit could also be chance alignments, but the probability drops for progressively smaller separations. In Upper Sco, we expect $~ 3.3$ chance alignments with separations of $15^{\prime \prime}-30^{\prime \prime}$ from an intermediate- or high-mass member $\left(M_{\text {prim }}>0.4 M_{\odot}\right)$, plus another $\sim 2.4$ chance alignments of two low-mass members. The number of high-mass chance alignments is far lower than the total number of pairs, which suggests that the vast majority are bound binaries. However, our wide binary survey found only four wide pairs of lowmass companions, so it is unclear whether any are genuine binary systems. The contamination rate is moderately lower in Taurus, yielding $\lesssim 1$ contaminant in either mass range, but the results are similar. Most of the high-mass pairs are binary systems, but the two low-mass pairs may or may not be bound binary systems.

A. L. K. is supported by a NASA Origins grant to L. A. H.

\section{REFERENCES}

Bate, M., Bonnell, I., \& Bromm, V. 2003, MNRAS, 339, 577

Bate, M., Clarke, C., \& McCaughrean, M. 1998, MNRAS, 297, 1163

Briceño, C., Luhman, K., Hartmann, L., Stauffer, J., \& Kirkpatrick, J. 2002, ApJ, 580, 317

Duchêne, G., Bouvier, J., Bontemps, S., André, P., \& Motte, F. 2004, A\&A, 427,651

Duquennoy, A., \& Mayor, M. 1991, A\&A, 248, 485

Frink, S., Roser, S., Neuhauser, R., \& Sterzik, M. 1997, A\&A, 325, 613

Fũrész, G., Hartmann, L., Megeath, S., Szentgyorgyi, A., \& Hamden, E. 2008, ApJ, 676, 1109

Goldsmith, P., Heyer, M., Narayanan, G., Snell, R., Li, D., \& Brunt, C. 2008, ApJ, 680, 428

Gomez, M., Hartmann, L., Kenyon, S., \& Hewett, R. 1993, AJ, 105, 1927

Hartmann, L. 2002, ApJ, 578, 914

Hewett, P. 1982, MNRAS, 201, 867

Jones, B., \& Herbig, G. 1979, AJ, 84, 1872

Kenyon, S., \& Hartmann, L. 1995, ApJS, 101, 117

Kraus, A., \& Hillenbrand, L. 2007a, ApJ, 662, 413 2007b, ApJ, 664, 1167
Kraus, A., \& Hillenbrand, L. 2008, ApJ, submitted

Lada, C., Margulis, M., \& Dearborn, D. 1984, ApJ, 285, 141

Larson, R. 1995, MNRAS, 272, 213

Luhman, K., Whitney, B., Meade, M., Babler, B., Indebetouw, R., Bracker, S., \& Churchwell, E. 2006, ApJ, 647, 1180

Mamajek, E. 2006, AJ, 132, 2198

Padgett, D., et al. 2006, BAAS, 209, 3016

Peebles, J. 1980, The Large Scale Structure of the Universe (Princeton: Princeton Univ. Press)

Preibisch, T., et al. 2002, AJ, 124, 404

Scelsi, L., et al. 2007, A\&A, 468, 405

Simon, M. 1997, ApJ, 482, L81

Slesnick, C., Carpenter, J., Hillenbrand, L., \& Mamajek, E. 2006, AJ, 132, 2665

Strom, K., \& Strom, S. 1994, ApJ, 424, 237

Webb, R., Zuckerman, B., Platais, I., Patience, J., White, R., Schwartz, M., \& McCarthy, C. 1999, ApJ, 512, L63

Williams, J., \& Garland, C. 2002, ApJ, 568, 259

Zuckerman, B., Song, I., \& Bessell, M. 2004, ApJ, 613, L65 